\title{
SN 2008iy circumstellar interaction: Bright and lesser light effects
}

\author{
N. N. Chugai ${ }^{\star}$ \\ ${ }^{1}$ Institute of Astronomy, Russian Academy of Sciences, Pyatnitskaya St. 48, 119017 Moscow, Russia
}

Accepted XXX. Received YYY; in original form ZZZ

\begin{abstract}
Optical photometry and spectra of the luminous type IIn supernova SN 2008iy are analysed in detail with implications for cosmic ray acceleration and the radio emission. The light curve and expansion velocities indicate ejecta with the kinetic energy of $3 \times 10^{51}$ erg to collide with the $\sim 10 M_{\odot}$ circumstellar envelope. The luminous $\mathrm{H} \alpha$ is explained as originated primarily

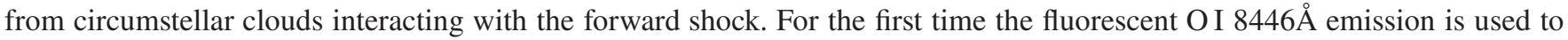
demonstrate that the cloud fragmentation cascade spans a scale range $>2.3$ dex. The narrow circumstellar $\mathrm{H} \alpha$ permitted us to estimate the acceleration efficiency of cosmic rays. The found value is close to the efficiency inferred in the same way for other two SNe IIn, SN 1997eg and SN 2002ic. The efficiency of cosmic ray acceleration is utilized to reproduce the radio flux from SN 2008iy for the amplified magnetic field consistent with the saturated turbulent magnetic field in the diffusive shock acceleration mechanism.
\end{abstract}

Key words: supernovae: general - supernovae: individual: SN 2008iy

\section{INTRODUCTION}

Type IIn supernovae are selected by the presence of narrow circumstellar lines in their spectra (Schlegel 1990). They generally reveal strong late time optical luminosity (Filippenko 1991; Stathakis \& Sadler 1991) powered by the ejecta interaction with the dense circumstellar (CS) matter (CSM) (Chugai 1990). This family is highly heterogeneous and includes at least two varieties: (i) SNe IIn interacting with a dense CS wind, e.g., SN $1988 Z$ (Stathakis \& Sadler 1991) and (ii) less numerous, SNe IIn interacting with a massive CS envelope presumably lost by a violent pulse ejection, e.g., SN 2006gy (Woosley et al. 2007; Smith \& McCray 2007). Noteworthy, SNe IIP and SNe IIL also show in early spectra (days-weeks) narrow CS emission lines (Khazov et al. 2016; Fassia et al. 2001) although it would be impractical to classify them as SNe IIn. Yet the physics behind narrow lines in early SNe IIP and SNe IIL has a lot in common with that of SNe IIn.

Not only the origin of SNe IIn is poorly understood, the physics of spectra formation in most cases is also far from clear. The interpretation of the optical phenomena of SNe IIn in terms of sphericallysymmetric model of SN/CSM interaction faces serious problems. The point is that emission lines in the spherical models are related to the swept-up shell between the forward and reverse shocks or/and outer unshocked ejecta, so predicted profiles are broad and top-hat, whereas observed profiles in most cases are more narrow and have "Gaussian" appearence. Supernova SN 1986J was first to demonstrate apparent mismatch between the high average expansion velocity of $1.3 \times 10^{4} \mathrm{~km} \mathrm{~s}^{-1}$ of the radioshell (Weiler et al. 1990) and the narrow $\mathrm{H} \alpha$ emission with FWHM of $\sim 1000 \mathrm{~km} \mathrm{~s}^{-1}$ (Rupen et al. 1987). The paradox is resolved under assumption that the $\mathrm{H} \alpha$ originates from shocked CS clouds (Chugai 1993). Archetypical SN $1988 Z$

^ E-mail: nchugai@inasan.ru with narrow, broad and intermediate $\mathrm{H} \alpha$ components (Filippenko 1991; Stathakis \& Sadler 1991) provides further evidence that the strong $\mathrm{H} \alpha$ intermediate component originates from shocked CS clouds (Chugai \& Danziger 1994).

Of particular significance for the SNe IIn understanding are events that are well observed photometrically and spectroscopically and discovered close to the explosion moment. This is the case of the luminous SN 2008iy that is prominent for the delayed 400-day rise towards broad light maximum and for the tremendous $\mathrm{H} \alpha$ luminosity of $\approx 7 \times 10^{41} \mathrm{erg} \mathrm{s}^{-1}$ (Miller et al. 2010), only 30\% lower than that of the record-holder SN 2010jl (Zhang et al. 2012). The photometric and spectral data of SN 2008iy have been interpreted in terms of the $\mathrm{SN}$ ejecta interaction with a dense clumpy wind lost by preSN a century before the explosion (Miller et al. 2010).

Miller et al. (2010) provide us with a guiding general picture of the phenomenon of SN 2008iy, yet some key issues escaped consideration. Of top interest are the total mass lost by preSN and the SN explosion energy. The similarity between SN 2008iy and SN 1988 Z emphasised by Miller et al. (2010) suggests that the H $\alpha$ of SN 2008iy might originate from shocked CS clouds. The modelling of $\mathrm{H} \alpha$ in this scenario would permit us to look at the profile asymmetry and check, whether a dust does form in the CDS and/or ejecta likewise in SN 2010j1 (Chugai 2018). Although the origin of the $\mathrm{H} \alpha$ emission from shocked clouds has no sensible alternative, one should emphasise the qualitative nature of this picture that leaves open some important issues. Particularly, a striking smoothenesss of the $\mathrm{H} \alpha$ profile in SN 2008iy (cf. Miller et al. 2010) raises a question, whether a random ensemble of shocked clouds is able to produce that profile?

The key implicit ingredient of the scenario with shocked clouds is their fragmentation and acceleration of fragments (Klein et al. 1994, 2003), which results in the formation of a broad velocity spectrum of $\mathrm{H} \alpha$-emitting gas constrained by the $\mathrm{H} \alpha$ profile (e.g. Chugai 2018). Given some arbitrariness of the described model one may ask our- 
selves, whether there is any independent observational evidence for the fragmentation of shocked CS clouds? It could be, e.g., a demonstration that we "see" significantly smaller line-emitting fragments compared to the size of original CS clouds. In this regard the O I $8446 \AA$ emission line in SN 2008iy spectra (Miller et al. 2010) may turn out an appropriate tool for probing small scale fragments. The point is that, if O I $8446 \AA$ line is of the fluorescent origin, which seems to be true, then we would be able to infer the $\mathrm{H} \alpha$ optical depth. The latter in turn depends on the size of $\mathrm{H} \alpha$-emitting fragments.

The high resolution Keck I/LRIS spectrum on day 711 after discovery shows narrow CS H $\alpha$ with a P Cyg profile (Miller et al. 2010). Qualitative analysis of this profile provides some hint that we may be see in this line the specific effect already met in the CS $\mathrm{H} \alpha$ of SN 1997eg and attributed to the preshock acceleration of the CS gas by the cosmic ray (CR) precursor (Chugai 2019).

The outlined issues strongly motivate us to revisit SN 2008iy and to look closer at at these interesting aspects of the phenomenon. The paper starts with the light curve modelling that provides us with the CS density distribution, the CSM mass, and the explosion energy. Thereafter I explore in detail the $\mathrm{H} \alpha$ emission including the origin of weak profile asymmetry, problem of profile smoothness, and parameters of line-emitting shocked clouds. The O I $8446 \AA$ emission will be used then to infer a size of the line-emitting fragments of shocked clouds. Finaly, the narrow CS $\mathrm{H} \alpha$ is analysed with important implications for the CR acceleration efficiency in SNe IIn and interpretation of the detected radio (Chandra \& Soderberg 2009).

\section{CS INTERACTION MODEL}

\subsection{Bolometric luminosity}

To reconstruct the bolometric light curve I rely on the light curve presented by $i$, and $I$ magnitudes (Miller et al. 2010) with two initial points in visual band and late three points in $r$ band. Miller et al. (2010) find that a bolometric correction can reach -1.2 for the $I$ band. Here I adopt BC = -1 mag for all the bands and recover the bolometric light curve with zero extinction correction, in line with Miller et al. (2010). The bolometric light curve is crucial for the modelling of the CS interaction and independent evidence in favour of the adopted bolometric correction is needed.

A strong support of this choice is provided by the high $\mathrm{H} \alpha$ luminosity. On day 652 from the discovery $(\sim 702 \mathrm{~d}$ after explosion) the bolometric luminosity recovered for $\mathrm{BC}=-1$ is $L_{b o l} \approx$ $1.2 \times 10^{43} \mathrm{erg} \mathrm{s}^{-1}$. The bolometric luminosity is expected to be very close to the unabsorbed X-ray luminosity of shocks. On the other hand, this X-ray luminosity can be recovered based on the $\mathrm{H} \alpha$ luminosity at this age $L(\mathrm{H} \alpha)=6.1 \times 10^{41} \mathrm{erg} \mathrm{s}^{-1}$ (Miller et al. 2010). This can be done as follows.

The $\mathrm{H} \alpha$ emission is powered by the absorbed X-rays. The deposited energy $\left(D \mathrm{erg} \mathrm{s}^{-1} \mathrm{~cm}^{-3}\right)$ in the form of fast photoelectrons is shared between the Coulomb heating, hydrogen ionization and excitation. With the energy fraction spent on the Coulomb heating $f_{h}$ the rate $\left(\mathrm{cm}^{-3} \mathrm{~s}^{-1}\right)$ of the hydrogen ionization and exitation of levels $n>2$ is approximated by the expression $(D / w)\left(1-f_{h}\right)$, where $w=22 \mathrm{eV}$ suggested by the expression of Xu et al. (1992). Assuming that all the recombinations and excitations on levels $n>2$ end up with the $\mathrm{H} \alpha$ emission one gets the $\mathrm{H} \alpha$ emissivity $\left(\mathrm{erg} \mathrm{cm}^{-3} \mathrm{~s}^{-1}\right.$ )

$\epsilon_{32}=\left(E_{23} / w\right)\left(1-f_{h}\right) D=\psi D$,

where $E_{23}=1.89 \mathrm{eV}$ is the energy of $\mathrm{H} \alpha$ photon. The numerical computations of $f_{h}(x)$ dependence on the ionization fraction
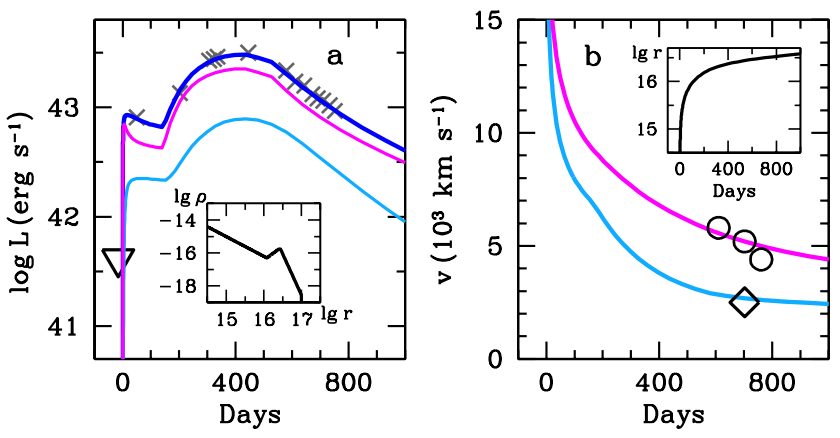

Figure 1. Left panel (a): bolometric light curve of SN 2008iy (crosses) and non-detection upper limit (triangle) with the model light curve for $8 M_{\odot}$ ejecta (blue) and contributions of the forward shock (magenta) and reverse shock (skyblue). Inset shows the CS density. Right panel (b): the model CDS velocity (skyblue) and ejecta velocity at the reverse shock (magenta); observational maximal ejecta velocity (Miller et al. 2010) are shown by circles, while the CDS velocity recovered from the $\mathrm{H} \alpha$ profile modelling is shown by diamond. Inset shows the CDS radius.
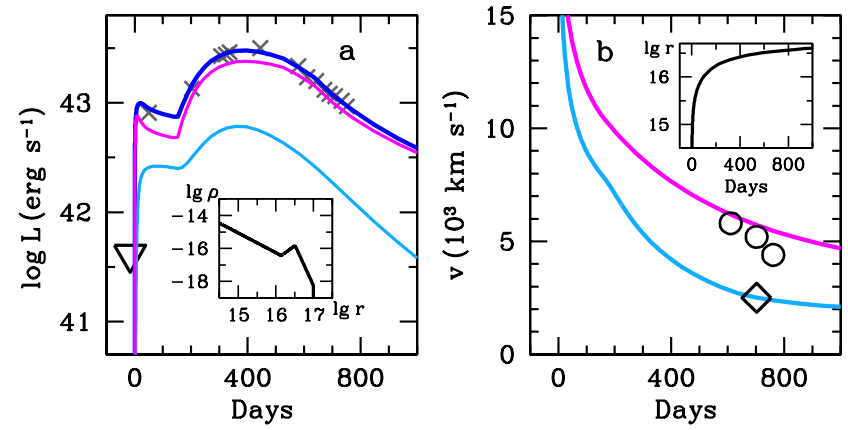

Figure 2. The same as Fig. 1 but for the $5 M_{\odot}$ ejecta.

(Xu et al. 1992) can be approximated as $f_{h}=x^{0.28}$ with the accuracy better than $10 \%$ in the range $0.01 \lesssim x<1$. For $0.05<x<0.1$ (cf. Section 3.5) $0.43<f_{h}<0.52$ and thus the $\mathrm{H} \alpha$ conversion efficiency $\psi \sim 0.044$. With this value the expected absorbed X-ray luminosity should be $\approx 1.35 \times 10^{43} \mathrm{erg} \mathrm{s}^{-1}$. This is close to the bolometric luminosity $\approx 1.2 \times 10^{43} \mathrm{erg} \mathrm{s}^{-1}$ assuming $\mathrm{BC}=-1 \mathrm{mag}$, which thus supports the adopted choice for the bolometric correction.

\subsection{Modelling interaction}

The exploded preSN could be a WR helium star with the radius of $\sim 10 R_{\odot}$ lost almost all the hydrogen envelope, or the preSN might retain significant amount of hydrogen envelope remaining a red supergiant (RSG) with a typical radius of $\sim 500 R_{\odot}$. The different preSN configuration however affects only initial part of the light curve $(t \lesssim 100 \mathrm{~d}$ ), so we consider only the case of the compact preSN that reminds us the case of SN 2001em (Chandra et al. 2020).

It is conceivable that the delayed light curve maximum might be related to the radiation diffusion in the massive CS envelope. This however would require a fully ionized $40 M_{\odot} \mathrm{CS}$ envelope. The latter in turn would suggest the presence of a narrow CS recombination $\mathrm{H} \alpha$ with the luminosity of $\sim 10^{43} \mathrm{erg} \mathrm{s}^{-1}$, which is at odds with SN 2008iy spectra. The diffusion mechanism for the delayed maximum therefore should be rejected. In line with Miller et al. (2010) the delayed maximum is considered as an outcome of SN ejecta interaction with the $\mathrm{CS}$ envelope at about $2 \times 10^{16} \mathrm{~cm}$. 
Table 1. Parameters of light curve models and output values on day 702

\begin{tabular}{|c|c|c|c|c|c|c|c|}
\hline $\begin{array}{l}M \\
M_{\odot}\end{array}$ & $\begin{array}{l}E \\
10^{51} \mathrm{erg}\end{array}$ & $\begin{array}{l}M_{c s}^{\dagger} \\
M_{\odot}\end{array}$ & $\begin{array}{l}v_{c d s} \\
\mathrm{~km} \mathrm{~s}^{-1}\end{array}$ & $\begin{array}{l}v_{s n} \\
\mathrm{~km} \mathrm{~s}^{-1}\end{array}$ & $\begin{array}{l}r_{c d s} \\
10^{16} \mathrm{~cm}\end{array}$ & $\begin{array}{l}\rho_{0} \\
10^{-17} \mathrm{~g} \mathrm{~cm}^{-3}\end{array}$ & $\begin{array}{l}M_{c d s} \\
M_{\odot}\end{array}$ \\
\hline 8 & 3.2 & 11.7 & 2700 & 5200 & 3.1 & 9.3 & 11 \\
\hline 5 & 3.2 & 12.7 & 2500 & 5700 & 3.6 & 10 & 11.6 \\
\hline
\end{tabular}

$\dagger$ Inside the radius of $4 \times 10^{16} \mathrm{~cm}$.

The CS interaction is modelled based on the thin shell approximation (e.g. Chugai 2018) that treats the swept up mass between the reverse and forward shock as a thin shell driven by ejecta dynamical pressure (Chevalier 1982b). Given the instant escape of the generated optical radiation, the shock radiative luminosity is adopted to be equal to the X-ray luminosity of both shocks, which in turn is calculated as the kinetic luminosity multiplied by the radiation efficiency $t /\left(t+t_{c}\right)$, where $t$ is the age and $t_{c}$ is the cooling time of the postshock gas. The latter is calculated based on the isothermal approximation $\left(T_{e}=T_{i}\right)$ with the postshock density being four times of the preshock density and the cooling function for the solar abundance (Sutherland \& Dopita 1993). The interaction model for the light curve assumes the average smooth CS density set by the broken power law, ignoring clumpiness that is a sound approximation for the probing CS mass and SN energy. For the explored parameter set both the reverse and forward shocks remain radiative untill day $\sim 700$. In reality, however, the forward shock in the intercloud gas is adiabatic.

The initial SN ejecta is set as the homologously expanding envelope $(v=r / t)$ with the density distribution $\rho=\rho_{0} /\left[1+\left(v / v_{0}\right)^{8}\right]$. Parameters $\rho_{0}$ and $v_{0}$ are defined via the ejecta mass $M$ and kinetic energy $E$. The radiation output of the ejecta/CSM interaction is determined by the kinetic energy of the ejecta external layers that can be similar for different combinations of the ejecta mass, kinetic energy, and CS density distribution. This means that to some degree there is a freedom for the ejecta mass, although constraints from the light curve and expansion velocities and conservative requirements of minimal kinetic energy and CS mass can bound the ejecta mass. The general rule is that the larger ejecta mass $>8 M_{\odot}$ requires higher kinetic energy, while lower ejecta mass $\left(<8 M_{\odot}\right)$ requires larger CS mass. We adopt the ejecta mass of $8 M_{\odot}$ for the fiducial model, but consider the case of $5 M_{\odot}$ ejecta as well.

Results for model A $\left(8 M_{\odot}\right)$ and model B $\left(5 M_{\odot}\right)$, assuming the explosion occured $50 \mathrm{~d}$ prior to the detection, are shown in Fig. 1 and Fig. 2, respectively, (for parameters see Table 1). The Table includes SN ejecta mass, kinetic energy, and the mass of the CSM in the range $r<4 \times 10^{16} \mathrm{~cm}$, and output values at the age $t=702 \mathrm{~d}$ : the CDS velocity, ejecta velocity at the reverse shock, CDS radius, preshock CS density, and the CDS mass (the total swept-up mass is by $\sim 1 M_{\odot}$ larger). The ${ }^{56} \mathrm{Ni}$ mass in both models is $0.07 M_{\odot}$ adopted to be similar to that of SN 1987A; the ${ }^{56} \mathrm{Ni}$ mass affects only the light curve minimum at about $100 \mathrm{~d}$. The initial light maximum is related to the diffusion cooling of ejecta calculated based on the Arnett (1980) approximation. Note that the forward shock dominates in the bolometric luminosity over the reverse shock. The recovered CS mass and density distribution are well constrained by the light curve and the ejecta velocity. This is demonstrated by the similar inferred CS mass for different ejecta mass (Table 1).

The major result of the interaction modelling is the large mass of the CS enevelope, $M_{C S} \sim 10 M_{\odot}$. The preshock density on day 702 in the model A of $9.3 \times 10^{-17} \mathrm{~g} \mathrm{~cm}^{-3}$ at the radius of $3.1 \times 10^{16} \mathrm{~cm}$ combined with the wind velocity of $45 \mathrm{~km} \mathrm{~s}^{-1}$ (Section 4) suggests the tremendous mass loss rate $\dot{M} \approx 0.09 M_{\odot} \mathrm{yr}^{-1}$ at about $200 \mathrm{yr}$ before the explosion.

\section{H $\alpha$ AND CS CLUMPINESS}

\subsection{Background}

The H $\alpha$ emission of SN 2008iy dominated by the intermediate component with the half width at half maximum of $\sim 1000 \mathrm{~km} \mathrm{~s}^{-1}$ presumably originates from CS clouds shocked in the forward shock likewise in the case of SN 1988 Z (Chugai \& Danziger 1994). Multidimensional computations of the blast wave interaction with an interstellar cloud (Klein et al. 1994, 2003) and laboratory experiments (Klein et al. 2003; Hansen et al. 2007) suggest the following qualitative picture.

The forward shock with the postshock speed $v_{p s} \approx v_{c d s}$ and density $\rho_{S}$ colliding with the CS cloud of the radius $a$ and density $\rho_{c}$ drives the transmitted (cloud) shock with the speed $v_{c} \approx$ $v_{p s}\left(\rho_{s} / \rho_{c}\right)^{0.5}$. The fast forward shock overtakes the cloud in the time $\sim a / v_{p s}$ and interacts with itself creating a backside high pressure that drives also the rear cloud shock. The cloud eventually gets crushed by both opposite shocks into the umbrella-shaped pancake that is rolled-up by shear flow into a vortex ring. The latter undergoes strong disruptive instabilities with the eventual turbulent distruction. The cloud crushing time is $t_{c c} \sim a / v_{c}$ and it takes $t_{f} \sim 4 \times t_{c c}$ for shear flow to roll-up the vertex ring, disrupt it into small fragments, and accelerate them to the speed comparable to $v_{p s}$. Finally, tiny fragments get mixed with the hot gas of the forward shock. The interaction with the clumpy CSM thus results in the two-phase structure of postshock layer with cold dense fragments in a broad range of scales and velocities imbedded into the hot gas. These cold fragments ionized by X-rays are responsible for the observed Gaussian-like $\mathrm{H} \alpha$ of SN 2008iy.

To reproduce the $\mathrm{H} \alpha$ emission by direct numerical simulations currently is beyond reach. The $\mathrm{H} \alpha$ formation in SN 2008iy therefore will be desribed below based on simplified models.

\subsection{Profile modelling}

The model that accounts for the $\mathrm{H} \alpha$ profile should include the emission of a random ensemble of shocked clouds and accelerated cloud fragments with some velocity spectrum. To cope with this complicated situation I assume the constant specific $\mathrm{H} \alpha$ emissivity and adopt the appropriate spherical mass-velocity distribution in the postshock layer between the forward shock and the CDS

$g(v)=\left\{\begin{aligned}\left(v / v_{1}\right)^{2} & \text { if } v<v_{1} \\ \left(v_{2}-v\right) /\left(v_{2}-v_{1}\right) & \text { if } v_{1}<v<v_{2} \\ 0 & \text { otherwise }\end{aligned}\right.$

where $v_{1}$ and $v_{2}$ are parameters determined by the $\mathrm{H} \alpha$ profile fit. The $v_{1}$ value is expectedly close to the cloud shock speed $v_{c}$, whereas the maximal velocity of accelerated cloud fragments $v_{2}<v_{c d s}$. 
The low velocities range $v<v_{1}$ qualitatively takes into account the emission from the cloud that is not yet fragmented and the vortex ring generation by the $\mathrm{KH}$ instability. The rotational velocities of the vortex combined with radial velocities result in the emegence of stripped cloud material with low velocities $v<v_{1}$ in the frame of the $\mathrm{SN}$ center.

Currently 3D-hydrodynamic simulations of the shock-cloud interaction are performed only for the adiabatic case. There is no, therefore, a relevant numerical example of the velocity spectrum for the ensemble of shocked CS clouds in the forward shock for the scenario we consider. The qualitative explanation of how this velocity spectrum in the range $v>v_{1}$ does form can be given in terms of the constant rate of CS clouds inflow into the forward shock, the cloud lifetime $t_{0}$, the cloud survival probability $p(t) \propto\left(1-t / t_{0}\right)$, and the steady acceleration of cloud fragments by the forward shock flow (Chugai 2018).

The radial coordinate of clouds and their fragments is assumed to be a random value in the shell $\Delta r$ between the CDS and the forward shock. The $\Delta r$ value is not defined in the thin shell model. One cannot apply also the self-similar solution for the driven adiabatic shock in which case $\Delta r$ is larger than for the radiative shock. We adopt $\Delta r / r_{c d s}=0.2$ compared, e.g., to $\Delta r / r_{c d s}=0.27$ in the adiabatic self-similar solution for the homologously expanding ejecta $\left(\rho \propto v^{-8}\right)$ in the steady wind $\left(\rho \propto r^{-2}\right)$ (Chevalier 1982a). The effect of the $\Delta r$ choice is explored a bit below.

On day 652 after discovery (post-explosion day 702) the $\mathrm{H} \alpha$ and the model calculated by the Monte Carlo (MC) technique are shown in Figure 3. The appropriate parameters of the velocity spectrum $g(v)$ are $v_{1}=950 \mathrm{~km} \mathrm{~s}^{-1}$ and $v_{2}=2300 \mathrm{~km} \mathrm{~s}^{-1}$. Shown are three versions: (a) without any continua absorption or scattering, (b) with the CDS optical depth $\tau_{c d s}=0.2$ produced possibly by dust or other absorbing agent; (c) with Thomson scattering in the unshocked ejecta $\left(\tau_{s n}=0.25\right)$ assuming homogeneous distribution of electron number density. The case with the Thomson scattering is preferred compared to other versions. We checked effect of zero albedo and found that the difference with the Thomson scattering is negligible, so formally the dust in ejecta is not rulled out. A possible additional contribution of the CDS combined with the Thomson scattering should be small, $\tau_{c d s}<0.1$. The uncertainty of the $\Delta r$ choice affects only the optimal value of the optical depth of unshocked ejecta. For $\Delta r / r_{c d s}=0.15$ and 0.25 the ejecta optical depth is 0.2 and 0.3 , respectively.

In the optimal case (c) the $\mathrm{H} \alpha$ is fully dominated by the emission of shocked clouds with only $1.6 \%$ contribution from unshocked ejecta, consistent with the Thomson optical depth, and $10 \%$ contribution from the CDS emission. In the latter case we admit the boxy $\mathrm{H} \alpha$ profile originated from CDS fragments produced by the RayleighTaylor instability of the CDS (e.g. Blondin \& Ellison 2001). The $\mathrm{H} \alpha$ luminosity on day 702 is of $6.1 \times 10^{41} \mathrm{erg} \mathrm{s}^{-1}$ (Miller et al. 2010) of which $5.4 \times 10^{41} \mathrm{erg} \mathrm{s}^{-1}$ (i.e. $88 \%$ ) is related to the shocked CS clouds.

The weak $\mathrm{H} \alpha$ blueshift suggests that the significant amount of dust is not formed at the considered age (702 d) neither in CDS nor in the ejecta. Basically, the dust is expected to form at $t>300$ days in the CDS (Pozzo et al. 2004) or ejecta. For example, in the well observed SN IIn 2010jl at the age $t \gtrsim 400 \mathrm{~d}$ the presence of the dust in ejecta and CDS is indicated by the significant $\mathrm{H} \alpha$ blueshift (Maeda et al. 2013; Chugai 2018). The dust absence in SN 2008iy on day 702 could be related to the unusually high late time luminosity and, therefore, high gas temperature that inhibits the dust formation. Indeed, with the CDS radius of $3.1 \times 10^{16} \mathrm{~cm}$ and the luminosity of $10^{43} \mathrm{erg} \mathrm{s}^{-1}$ the effective temperature at this age is $\approx 2000 \mathrm{~K}$. This value slightly exceeds the condensation temperature of the graphite $(1800 \mathrm{~K})(\mathrm{e} . \mathrm{g}$.

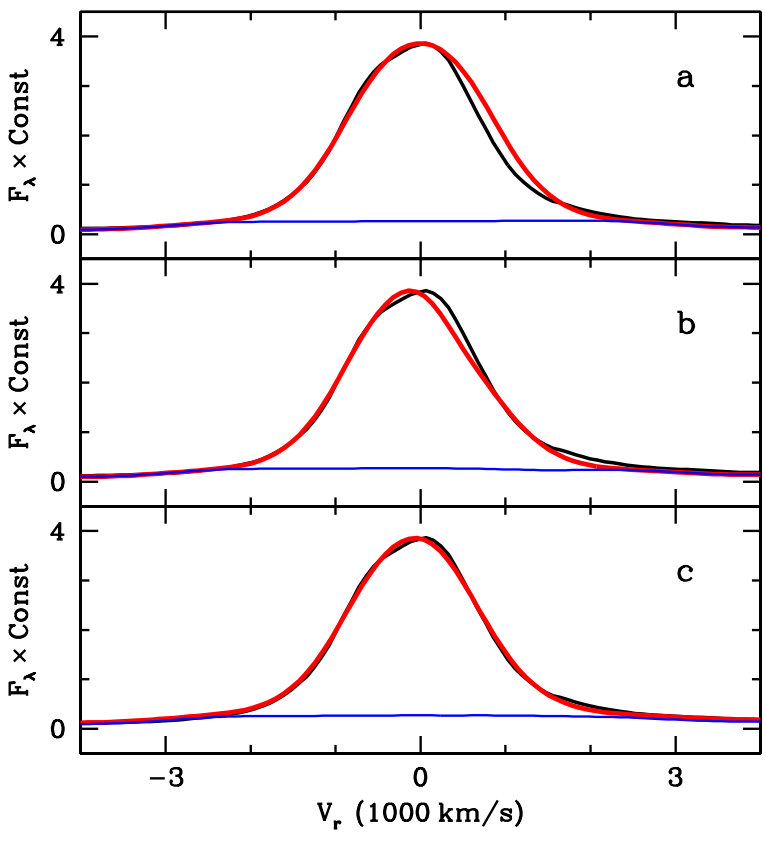

Figure 3. The $\mathrm{H} \alpha$ line on day 652 after discovery (black) with model profiles: $\mathbf{a}$ - without any continuum absorption/scattering, $\mathbf{b}$ - with the continuum absorption in the CDS, $\mathbf{c}$ - with the Thomson scattering in the unshocked ejecta.

Kozasa et al. 1989) that is the maximal among astrophysical dust species.

Yet the CDS with the column density of $2 \mathrm{~g} \mathrm{~cm}^{-2}$ and the temperature of $\sim 2000 \mathrm{~K}$ could contain significant amount of $\mathrm{H}^{-}$molecules, which are efficient absorbers in the $\mathrm{H} \alpha$ band. To estimate the optical depth produced by $\mathrm{H}^{-}$in the model A we use the CDS density suggested by the upstrean ram pressure $\rho=\rho_{0}\left(v_{s} / c_{s}\right)^{2}=$ $2.4 \times 10^{-11} \mathrm{~g} \mathrm{~cm}^{-3}$, where $c_{S} \approx 5.2 \mathrm{~km} \mathrm{~s}^{-1}$ is the sound speed of the CDS gas. We adopt the presense of $1 M_{\odot}$ of hydrogen in ejecta, whereas in the CSM the hydrogen abundance is assumed to be 0.7 . The electron number density is calculated for the absorbed X-ray luminosity of $10^{43} \mathrm{erg} \mathrm{s}^{-1}$ deposited in the CDS on day 700, while the number density of $\mathrm{H}^{-}$is determined by the Saha equation. The inferred CDS optical depth due to $\mathrm{H}^{-}$is $\tau_{c d s}=0.08$ that is marginally consistent with the upper limit $(<0.1)$ imposed by the $\mathrm{H} \alpha$ modelling.

\subsection{H $\alpha$-emitting clouds and fragments}

\subsubsection{Cloud size and density}

The undisturbed cloud density $\rho_{c}$ is related to the average preshock CS density $\rho_{0}$, cloud filling factor of undisturbed CS clouds $f_{0}$, and the intercloud mass fraction $\phi$ as

$\rho_{c}=\rho_{0}(1-\phi) f_{0}^{-1}$,

In turn, the cloud shock with the velocity $v_{c} \approx 900 \mathrm{~km} \mathrm{~s}^{-1}$ is driven by the postshock flow with the density $\sim 4 \rho_{0}$ and velocity $v_{p s}=$ $2700 \mathrm{~km} \mathrm{~s}^{-1}$ on day 702 , so that $v_{c}=v_{p s}\left(4 \rho_{0} / \rho_{c}\right)^{1 / 2}$ and therefore $\rho_{c} / \rho_{0}=4\left(v_{p s} / v_{c}\right)^{2} \approx 36$. Note, for the forward postshock density we use the average, and not intercloud density. Doing in this way we take into account fragmentation and mixing of tiny fragments of shocked clouds with the shocked intercloud gas. Following the similar case of SN 1997eg (Chugai 2019) we adopt here $\phi=0.5$. These values along with the Equation (2) result in $f_{0}=0.014$. For 
the average density $\rho_{0} \approx 9 \times 10^{-17} \mathrm{~g} \mathrm{~cm}^{-3}$ on day 702 (Table 1 ) the unperturbed cloud density is thus $\rho_{c}=3 \times 10^{-15} \mathrm{~g} \mathrm{~cm}^{-3}$ with the hydrogen number density $n_{c}=1.3 \times 10^{9} \mathrm{~cm}^{-3}$ assuming the hydrogen abundance $X=0.7$, while for $\phi=0.5$ the intercloud density is $n_{i c}=n_{c} f_{0} /\left(1-f_{0}\right)=2.5 \times 10^{7} \mathrm{~cm}^{-3}$.

The cooling time of the cloud shock is

$t_{\text {cool }} \approx \frac{m_{p} v_{c}^{2}}{32 n_{c} \Lambda} \approx 10^{4} \mathrm{~s}$,

where the cooling function $\Lambda=3 \times 10^{-23} \mathrm{erg} \mathrm{s}^{-1} \mathrm{~cm}^{3}$ for $T=10^{7} \mathrm{~K}$ (Sutherland \& Dopita 1993). The condition that the cooling time should be less than the cloud crushing time $t_{c c}=a / v_{c}$ implies the lower limit of the cloud radius $a>v_{c} t_{\text {cool }} \approx 10^{12} \mathrm{~cm}$.

The upper limit of $a$ is determined by the condition that the cloud fragmentation time $t_{f} \sim 4 t_{c c}$ should be less than the time it takes for the forward shock to advance by the distance $\Delta r=0.2 r_{c d s}$, i.e., $t_{f}<\Delta r / v_{p s}$, which results in $a<(\Delta r / 4)\left(v_{c} / v_{p s}\right) \approx 5 \times 10^{14} \mathrm{~cm}$. This upper limit imposes a constraint on the number of CS clouds $\left(N_{c}\right)$ in the layer between the forward shock and the CDS from the obvious equality $(4 \pi / 3) a^{3} N_{c}=f_{0} V$, where $V=9 \times 10^{49} \mathrm{~cm}^{3}$ is the layer volume. The inequality $a<5 \times 10^{14} \mathrm{~cm}$ then implies the lower limit $N_{c}>10^{3}$.

The recombination $\mathrm{H} \alpha$ luminosity from shocked clouds and fragments in the forward shock layer $\Delta r=0.2 r_{c d s}$ with the volume $V=9 \times 10^{49} \mathrm{~cm}^{3}$ on day 702 is $L_{32}=\alpha_{32} n_{e}^{2} V f E_{23}$, where $f \ll f_{0}$ is the filling factor of the line-emitting fragments, $E_{23}$ is $\mathrm{H} \alpha$ photon energy, $\alpha_{32}=1.8 \times 10^{-13} \mathrm{~cm}^{3} \mathrm{~s}^{-1}$ is the $\mathrm{H} \alpha$ effective recombination coefficient in the recombination case $\mathrm{C}$ (opaque Balmer lines) for $T_{e}=10^{4} \mathrm{~K}$. For the $\mathrm{H} \alpha$ luminosity of the shocked clouds on day 702 of $5.4 \times 10^{41} \mathrm{erg} \mathrm{s}^{-1}$ the electron number density is therefore $n_{e}=8 \times 10^{8} \eta^{1 / 2} \mathrm{~cm}^{-3}$ where $\eta=f_{0} / f$ is the compression factor that exceeds adiabatic value $(\eta=4)$ due to the radiative cooling and isobaric compression of the shocked gas. The upper limit for the compression factor in the radiative cloud shock is $\eta=\left(v_{c} / c_{s}\right)^{2} \sim 10^{4}$, where $c_{s} \sim 10 \mathrm{~km} \mathrm{~s}^{-1}$ is the sound speed in $\mathrm{H} \alpha$-emitting gas. However the real value of $\eta$ can be lower and will be estimated in Section 3.5 .

\subsubsection{Ho smootheness}

The notable smootheness of the $\mathrm{H} \alpha$ line on day 711 (Fig. 4) with the flux fluctuation at the velocity scale of $\sim 200 \mathrm{~km} \mathrm{~s}^{-1}$ of $\lesssim 1 \%$ is challenging problem for the scenario of clumpy line-emitting zone. Obviously, the number of line-emitting fragments of shocked clouds should be large enough to significantly reduce a fluctuation amplitude.

The minimal number of cloud fragments imposed by the low amplitude of flux fluctuations can be estimated via MC simulations. We consider here the emission of shocked CS clouds omitting small contribution of unshocked ejecta and CDS. Generally, one has to distiguish between two populations of line-emitting clouds: nonfragmented clouds (simply "clouds") with the number of $N_{c}$ and their numerous fragments $\left(N_{f} \gg N_{c}\right)$. Here we consider the simplified set up assuming that both clouds and fragments has the same luminosity weight $w=1$, while their velocity are diced according to the distribution $g(v)$. The cloud position on the sphere is random. The resulting profile is then convolved with the Gaussian filter adopting the resolution of $100 \mathrm{~km} \mathrm{~s}^{-1}$.

The effect of fragment number is demonstrated in Figure 5 for $N_{f}=10^{4}$ and $10^{6}$. Only in the case of $N_{f}=10^{6}$ fluctuations become comparable to those observed on day 711. At first glance this

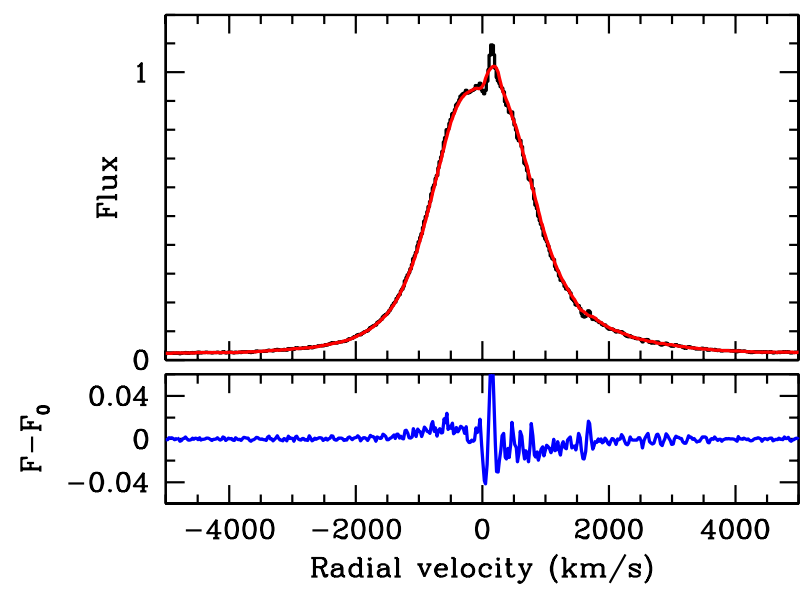

Figure 4. $\mathrm{H} \alpha$ in SN 2008 spectrum on day 711 after discovery (black) with overploted smoothed version. The lower panel shows residual between spectrum and smoothed version to demonstrate the fluctuations related to the clumpiness of the line-emitting gas. Fluctuation amplitudes ralated to clumpiness (if any) do not exceed $1 \%$. Narrow CS $\mathrm{H} \alpha$ produces in the residual prominent feature at zero velocity. The observational profile is shifted toward the red by $120 \mathrm{~km} \mathrm{~s}^{-1}$ in order to compensate the blueward skewing related to the ejecta scattering (absorption).

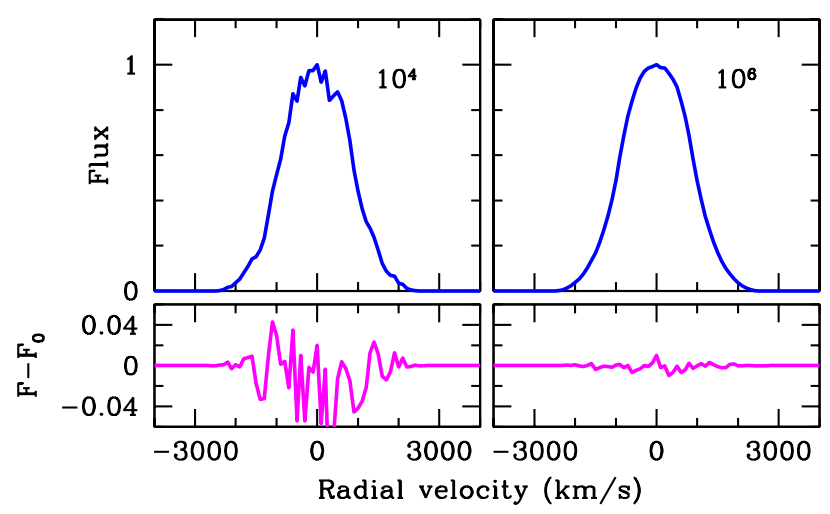

Figure 5. Monte Carlo simulation of $\mathrm{H} \alpha$ line emission produced by superposition of random line-emitting fragments of shocked clouds. Shown are cases with $N_{f}=10^{4}$ (left) and $10^{6}$ (right). Lower panels show residual with respect to the case of $10^{8}$ fragments. The fluctuations at the level $\lesssim 1 \%$ require $N_{f} \gtrsim 10^{6}$.

number is unrealisticaly large. In fact not, because to provide $10^{3}$ fold increase of fragments number compared to the shocked clouds $\left(\sim 10^{3}\right)$ the cloud of the radius $a$ should break up into cloudlets with the radius of only $0.1 \times a$. As will be shown below (Section 3.5) the cloud fragmentation cascade spans the scale range exceeding 2.3 dex, which implies that $N_{f}>10^{6}$.

Summing up, the smootheness of $\mathrm{H} \alpha$ is simply an outcome of the large number of line-emitting cloud fragments $\left(>10^{6}\right)$ in the forward shock. Noteworthy that the scenario of the line emitting fragments predicts the maximal fluctuation amplitude in the low velocity range $\left(\left|v_{r}\right|<1500 \mathrm{~km} \mathrm{~s}^{-1}\right.$ ) of the profile (Fig. 5), which seems to be consistent with the picture of fluctuations in the observed $\mathrm{H} \alpha$ (Fig. 4). 


\subsection{Hydrogen ionization}

We already saw (Section 2.1) that to account for the $\mathrm{H} \alpha$ luminosity at the age of $702 \mathrm{~d}$ the line-emitting gas should absorb X-rays with the luminosity of $\sim 10^{43} \mathrm{erg} \mathrm{s}^{-1}$ comparble to the bolometric luminosity. The hydrogen ionization of shocked CS clouds can be maintained by three sources: (i) X-rays of the reverse and forward shock, (ii) X-rays of cloud shocks, and (iii) XUV radiation from a hot boundary layer at the interface between the cold dense gas of shocked clouds and the hot gas of the forward shock. The X-rays from the reverse shock are significantly absorbed by the CDS in the range $h v<4.5 \mathrm{keV}$ and given low luminosity of the revese shock it does not contribute significantly to the ionization of shocked clouds.

For the X-ray of the forward shock to be efficient ionizing agent of shocked clouds their surface area $S$ should provide efficient covering, i.e., their area ratio $A=S / 4 \pi r_{c d s}^{2}$ should be greater than unity. If this is the case, the radial hydrogen column density determined by the total CS cloud mass $\rho_{0} V(1-\phi)$ is $N_{\mathrm{H}} \sim 1.3 \times 10^{23} \mathrm{~cm}^{-2}$. This value implies the efficient absorption of X-rays in the range $h v<4 \mathrm{keV}$.

$\mathrm{X}$-rays of cloud shocks with the temperature of $\sim 1 \mathrm{keV}$ provide the luminosity $L_{c}=2 \pi C r_{c d s}^{2} \rho_{c} v_{c}^{3}=1.5 \times 10^{43} C \mathrm{erg} \mathrm{s}^{-1}$, where $C$ is the covering factor of shocked clouds, $C=(3 / 2)\left(f_{0} V / a\right) /\left(4 \pi r_{c d s}^{2}\right) \gtrsim$ 0.3 , since $a<5 \times 10^{14} \mathrm{~cm}$. This means that X-ray emission of shocked CS clouds is able to significantly contribute to the hydrogen ionization of shocked CS clouds.

The hydrogen of cold fragments could be ionized also via the electron conductivity flux from the background hot plasma of the forward shock ( $T \approx 8 \mathrm{keV}$ ). The conductivity heating of cold dense fragments is impeeded by the magnetic field of dense fragments. Yet a hybrid mechnism could operate that includes an extreme UV radiation dominanted by He II $304 \AA$ from a hot dense boundary layer at the interface between cold fragments and hot gas of the forward shock. The heat flux can be written as a fraction $\zeta$ of the saturation flux $F_{\text {sat }}=0.2 n k T_{e} u_{e}$ (Cowie \& McKee 1977), where $u_{e}$ is the electron mean thermal velocity. For $T_{e}=8 \mathrm{keV}$ and $n=$ $4 n_{i c}=10^{8} \mathrm{~cm}^{-3}$ one gets $F_{\text {sat }}=2 \times 10^{9} \mathrm{erg} \mathrm{s}^{-1} \mathrm{~cm}^{-2}$. The area of the interface between hot and cold phases is $S \sim 10^{35}(A / 10) \mathrm{cm}^{2}$, which implies the conductive luminosity $L_{\text {con }}=\zeta F_{\text {sat }} S=2 \times$ $10^{44}(A / 10) \zeta \mathrm{erg} \mathrm{s}^{-1}$. Even for $\zeta \sim 0.1$, the heat conductivity meets the requirement for the $\mathrm{H} \alpha$ energizing.

We thus find ourselves in a situation when all three sources (Xrays from the forward shock, X-rays from shocked clouds, thermal conductivity) can efficiently contribute to the hydrogen ionization of shocked clouds. At the moment we are not able to conclude, which of these mechanisms dominates.

\subsection{O I $8446 \AA$ and cloud fragmentation}

\subsubsection{Ho optical depth}

Between days 560 and 652 after discovery SN 2008iy spectra show O I $8446 \AA$ emission line (Miller et al. 2010) at the blue side of the Ca II $8600 \AA$ triplet. The absence of the similar O I $7774 \AA$ emission indicates that the $8446 \AA$ emission cannot be produced by the oxygen recombination or collisional excitation in which case the intensity of both lines whould be comparable (cf. Borkowski \& Shull 1990). The likely origin of O I $8446 \AA$ is therefore the fluorescence (Bowen 1947) due to the absorption of $\mathrm{L} \beta 1025.728 \AA$ in the ultraviolet transition $2 \mathrm{p}^{3} \mathrm{P}-3 \mathrm{~d}^{3} \mathrm{D}^{\circ}$ of O I $1025.762 \AA$. Noteworthy, Leibundgut et al. (1991) have identified O I $8446 \AA$ emission in the spectrum of SN 1986J and suggested its fluorescent origin.

The O I $8446 \AA$ line profile on day 652 recovered by deblending

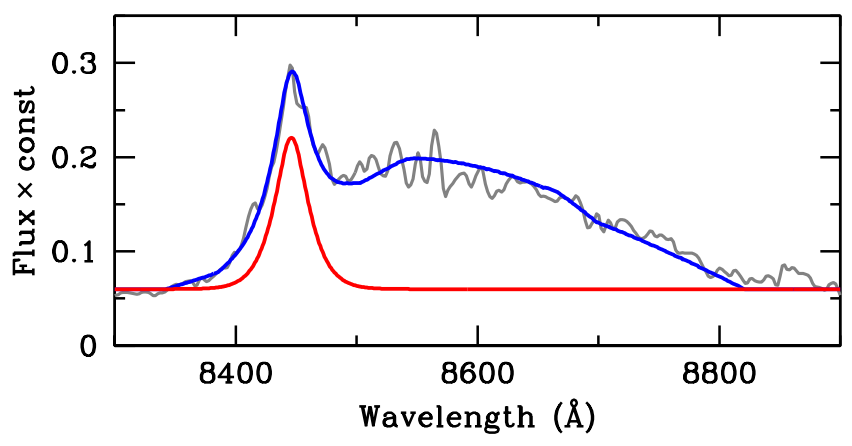

Figure 6. O I $8446 \AA$ emission blended with Ca II triplet in the SN 2008iy spectrum on day 652. Red line shows deblended contribution of O I $8446 \AA$. Left panel shows the case of similar line profile for O I line and Ca II lines, which indicates that $\mathrm{Ca}$ II lines should be broader. Right panel shows the case of broader Ca II lines. Inset shows the normalized emissivity distribution for O I line (red) and Ca II lines in this case.

(Fig. 6) suggests the flux ratio $R=F(8446) / F(\mathrm{H} \alpha)=0.05$. This ratio depends on the $\mathrm{H} \alpha$ optical depth $\tau_{23}$ (Netzer \& Penston 1976) and thus can be used to estimate the hydrogen excitation degree. Note the obvious difference of the profiles of O I and Ca II lines (Fig. 6), which reflects a different origin of fluorescent O I line and collisionally excited $\mathrm{Ca}$ II lines.

The $\mathrm{L} \beta$ photon emitted with the branching ratio $p_{1}=0.56$ can be absorbed by the oxygen with the probability $p_{2}$ that depends on the oscilator strength of corresponding $\mathrm{H}$ and $\mathrm{OI}$ transitions and oxygen abundance. Note that the hydrogen and oxygen are mostly neutral in the shocked clouds where $\mathrm{H} \alpha$ and O I $8446 \AA$ presumably originate from. Assuming the solar $\mathrm{O} / \mathrm{H}$ ratio $\left(6 \times 10^{-4}\right.$ by number $)$ and using NIST data for oscilator strengths of both transitions one finds $p_{2}=8.4 \times 10^{-5}$. The $\mathrm{L} \beta$ absorption by the oxygen is followed by the cascade of $11287 \AA$ and $8446 \AA$ lines with the branching ratio $p_{3}=0.3$. The exitation of the third hydrogen level thus ends up by the emission of O I $8446 \AA$ photon with the probability $p_{31}=p_{1} p_{2} p_{3}=$ $1.41 \times 10^{-5}$. The flux ratio of O I $8446 \AA$ and $\mathrm{H} \alpha$ is then

$R=\frac{A_{31} p_{31}}{A_{32} \beta_{23}}\left(\frac{6563}{8446}\right)=1.38 \times 10^{-5} \beta_{23}^{-1}$,

where $\beta_{23}$ is the $\mathrm{H} \alpha$ escape probability. For the observational value $R=0.05$ the Equation (4) gives $\beta_{23}=2.8 \times 10^{-4}$ or the average number of $\mathrm{H} \alpha$ photon scattering $N_{S}=1 / \beta_{23}=3.6 \times 10^{3}$.

The fragments of the dirupted cloud have an intricate geometry (e.g., Klein et al. 2003) that can be approximately viewed as a combination of sheets and filaments. The cumulative surface $S$ of fragments is described by their number $N$, the minimal $h$ and maximal $l$ scales of typical fragment as $S \sim N l^{2}$ for sheets and $S \sim N l h$ for filaments. Noteworthy that the expression for the total volume of sheets and flaments is the same $S h=f V$, i.e., independent of the fragments geometry. This expression establishes the useful relation between $h$ and $S$, or area ratio $A$ for a given $f$ and CDS radius $r_{c d s}$.

To estimate the $\mathrm{H} \alpha$ optical depth from the number of scatterings we approximate a typical fragment by a plane slab with the central $\mathrm{H} \alpha$ optical depth $\tau_{23}$. For the homogeneous density and excitation rate in the slab the $\mathrm{H} \alpha$ raiation transfer with the Doppler absorption coefficient and the complete frequency redistribution results in the average number of scattering

$N_{s}=Z \tau_{23} \sqrt{\pi \ln \tau_{23}}$,

with $Z \sim 1$ (Capriotti 1965). Our direct MC simulation of double diffusion (space and frequency) results in $Z=0.46$ in the range of 
$1000<\tau_{23}<2000$. Given the observational estimate of $N_{S}$ one gets then $\tau_{23}=1.6 \times 10^{3}$.

\subsubsection{Evidence for cloud fragmentation}

The $\mathrm{H} \alpha$ optical depth combined with the luminosity of $\mathrm{H} \alpha$ emitted by shocked clouds permit us to determine two parameters related to the $\mathrm{H} \alpha$-emitting fragments of shocked clouds - compression factor $\eta$ and the minimal size $h$. This can be done via modelling the hydrogen ionization and excitation.

Equations of the statistcal equilibrium for two-level plus continuum hydrogen atom are solved for the electron number density $n_{e}=8 \times 10^{8} \eta^{1 / 2}$ suggested by the $\mathrm{H} \alpha$ luminosity on day 702 , the hydrogen number density $n=1.3 \times 10^{9} \eta$ at the same age, and the deposition rate of the absorbed X-rays $D=L(\mathrm{H} \alpha) /(\psi f V)$, imposed by $\mathrm{H} \alpha$ luminosity $L\left(\mathrm{H} \alpha=5.4 \times 10^{41} \mathrm{erg} \mathrm{s}^{-1}\right.$, with the conversion efficiency $\psi$ defined by the Equation (1), the filling factor $f=f_{0} / \eta$, and the volume of the forward shock layer $V=9 \times 10^{49} \mathrm{~cm}^{3}$. Apart from the non-thermal excitation and ionization the model includes collisional transitions, recombinations on levels $n \geqslant 2$ that eventually populate the second level, the two-photon decay, and L $\alpha$ scattering.

The statistcal equilibrium is calculated in the temperature range $T_{e} \geqslant 7000 \mathrm{~K}$. The law limit is set based on the fact that the cooling function for $T_{e}=7000$ is $3 \times 10^{-25} \mathrm{erg} \mathrm{s}^{-1} \mathrm{~cm}^{3}$, i.e. by 2 dex lower than at the cloud shock, and rapidly drops for the decreasing temperature (Judge \& Neff 1990). In fact, even $7000 \mathrm{~K}$ is already unattainable, but we include this value for generality. Constraints imposed by the $\mathrm{H} \alpha$ optical depth $\tau_{23}=1.6 \times 10^{3}$ and $\mathrm{H} \alpha$ luminosity suggest solutions for $T_{e}=7000 \mathrm{~K}, 8000 \mathrm{~K}$, and $9000 \mathrm{~K}$ as follows $(\eta, A, x)=(51,400,0.09),(58,450,0.08)$, and $(460,800,0.03)$. Taking into account that $f=f_{0} / \eta$ one gets for $T_{e}>7000 \mathrm{~K}$ the minimal length scale of fragments $h=f V /\left(4 \pi r_{c d s}^{2} A\right)<5 \times 10^{9} \mathrm{~cm}$.

The found size of line-emitting fragments is by more than 2.3 dex lower compared to the lower limit of the cloud radius $10^{12} \mathrm{~cm}$ inferred from the condition of fast cooling in the cloud shock (Section 3.3). This is the direct outcome of the fragmentation cascade accompanying the disruption of the shocked CS clouds in the forward shock. It is easily to show that for the Kolmogorov turbulence the complete fragmentation cascade takes the time comparable to the fragmentation time of the largest scale, which in our case is of the order of the cloud crushing time $t_{c c} \sim a / v_{c}$.

\section{NARROW H $\alpha$ AND CR ACCELERATION}

\subsubsection{Modelling narrow $\mathrm{H \alpha}$}

The high-resolution Keck spectrum on day 711 day after discovery shows, on top of the intermediate component, the narrow $\mathrm{H} \alpha$ with the P Cyg profile that apart from the scattering contains also the net emission with the luminosity of $(2.8 \pm 0.6) \times 10^{39} \mathrm{erg} \mathrm{s}^{-1}$ (Miller et al. 2010). In the model A ( $8 M_{\odot}$ ejecta) on day 761 the volume emission measure of the CSM outside the shock $\left(>3.3 \times 10^{16} \mathrm{~cm}\right)$ in terms of smooth density and full ionization is $E M=1.5 \times 10^{64} \mathrm{~cm}^{-3}$. With the $\mathrm{H} \alpha$ effective recombination coefficient for $T_{e}=10000 \mathrm{~K}$ of $1.18 \times$ $10^{-13} \mathrm{~s}^{-1} \mathrm{~cm}^{3}$ (Martin 1988) one gets the luminosity $L(\mathrm{H} \alpha) \approx 5.3 \times$ $10^{39} \mathrm{erg} \mathrm{s}^{-1}$. Although simple in approach, this estimate indicates that the refined model with the $\mathrm{H} \alpha$ originated from partially ionized clouds could be closer to the observational luminosity of narrow $\mathrm{H} \alpha$. Below we model the narrow $\mathrm{H} \alpha$ in terms of a relative flux.

First, one should to pinpoint the rest frame. Narrow CS emission lines of [O I] 6300.30, 6363.78 A doublet in the spectrum on day 711

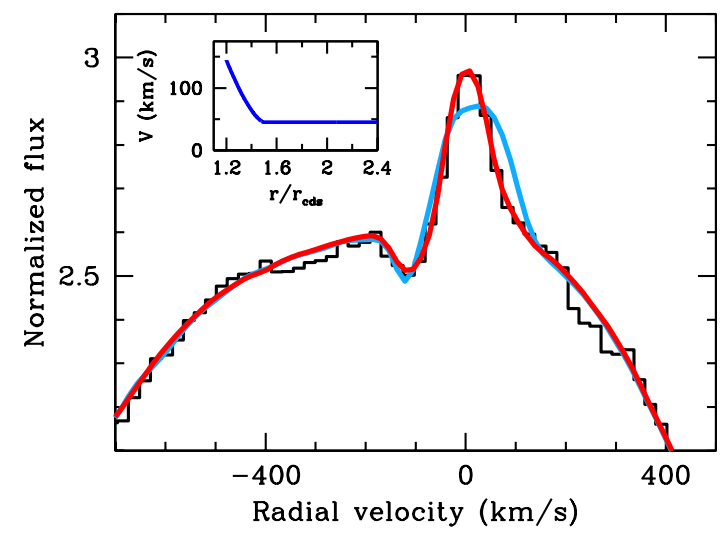

Figure 7. Narrow $\mathrm{H} \alpha$ in the Keck I spectrum on day 711 after discovery (black) with the overplotted optimal model (red). The model suggests the preaccelerated CSM with the speed at the forward shock of $145 \mathrm{~km} \mathrm{~s}^{-1}$. Inset shows the velocity of the CSM with the preaccelerated inner layer. The alternative model with the constant wind speed of $120 \mathrm{~km} \mathrm{~s}^{-1}$ (skyblue) is able to describe the absorption component but cannot reproduce the emission component.

show additional redshift of $+25 \pm 5 \mathrm{~km} \mathrm{~s}^{-1}$ compared to $z=0.0411$. This small correction is implemented in the rest frame wavelength in Figure 7; the excellent match between the observed emission peak and the model supports the correction.

The modelling of the narrow $\mathrm{H} \alpha$ indicates that the observed profile cannot be reproduced assuming the constant wind velocity along the radius that is demonstrated for the case of the constant CSM velocity of $120 \mathrm{~km} \mathrm{~s}^{-1}$ (Fig. 7). While the absorption component can be described in this scenario, the emission component turnes out to be unacceptably broad. The appropriate model (Fig. 7) includes the outer wind with the velocity of $45 \mathrm{~km} \mathrm{~s}^{-1}$ and the preshock accelerated wind with the velocity decreasing outward from $145 \mathrm{~km} \mathrm{~s}^{-1}$ at the shock $\left(r_{s}=1.2 r_{c d s}\right)$ down to $45 \mathrm{~km} \mathrm{~s}^{-1}$ at the radius $r=1.5 r_{c d s}$. The velocity of the accelerated wind is constrained by the blue absorption wing, whereas the outer wind velocity is constrained by the narrow upper part of the CS emission component. The Keck spectrum on day 711 shows narrow coronal line [Fe X] $6374 \AA$ (Miller et al. 2010) that presumably originates from the hot intercloud medium photoionized by X-rays of the forward shock likewise in SN 1997eg (Chugai 2019). The narrow $\mathrm{H} \alpha$ therefore likely originates from the cloudy component of the CSM. The depth of the absorption component suggests that the clouds filling factor $f_{c} \geqslant 0.05$; the case $f_{c}=0.05$ is shown in Figure 7. This value exceeds the earlier estimate $f_{0}=0.014$ (Section 3.3.1). The contradiction can be reconciled, if clouds have a core and a rarefied halo with the radius by a factor of 1.5 larger than that of the core.

Noteworthy, to reproduce the asymmetry of the broad (intermediate) component on day 761 (Fig. 7) the continuum optical depth of the CDS combined with the unshocked ejects should be 0.55 , compared to the optical depth 0.25 on day 702 . This indicates that the dust probably starts to form. The conjecture is supported by the effective temperature at this stage $(1700 \mathrm{~K})$ that is slightly lower than the graphite condensation temperature $(1800 \mathrm{~K})$.

\subsubsection{CR precursor}

The preshock CSM acceleration implied by the narrow $\mathrm{H} \alpha$ has been invoked earlier to account for the similar CS H $\alpha$ in SN 1997eg (IIn) on day 198 in which case it is atributed to the CR precursor 
Table 2. CR acceleration efficiency for SNe IIn

\begin{tabular}{lccccc}
\hline SN & Day & $u^{\dagger}$ & $u_{\max }$ & $v_{S}$ & $\epsilon$ \\
\hline 2008iy & 761 & 45 & 145 & 2600 & 0.038 \\
2002ic & 256 & 80 & 250 & 6000 & 0.028 \\
1997eg & 198 & 20 & 160 & 5000 & 0.028 \\
\hline
\end{tabular}

$\dagger$ Velocity in $\mathrm{km} \mathrm{s}^{-1}$.

(Chugai 2019). The CR are presumably accelerated by the standard mechanism of the diffusive shock acceleration (DSA) (Axford et al. 1977; Krymskii 1977). The CR pressure $\left(p_{c}\right)$ at the forward shock with respect to the upstream ram pressure $\left(\rho_{0} v_{s}^{2}\right)$ can be estimated using the undisturbed CS velocity $(u)$, preshock CS velocity $\left(u_{\max }\right)$ and the shock speed $\left(v_{S}\right)$ based on the equation of the momentum conservation

$\rho v \frac{\partial v}{\partial x}+\frac{\partial p_{c}}{\partial x}=0$

where I neglect the upstream gas pressure. Along with the mass conservation, the integration in the upstream flow in the shock frame with the boundary conditions $v(-\infty)=v_{s}-u, p_{c}(-\infty)=0$ and $v(0)=v_{s}-u_{\max }, p_{c}(0)=p_{c}$ results in the efficiency of $\mathrm{CR}$ acceleration

$\epsilon \equiv p_{c} / \rho_{0} v_{s}^{2}=\left(u_{\max }-u\right) / v_{s}$,

where small quadratic terms are omitted. With $u_{\max }=145 \mathrm{~km} \mathrm{~s}^{-1}$, $u=45 \mathrm{~km} \mathrm{~s}^{-1}$ and $v_{s}=2600 \mathrm{~km} \mathrm{~s}^{-1}$ at $t=761 \mathrm{~d}$ the efficiency of CR acceleration is then $\epsilon=0.038$.

The late high resolution spectra of $\mathrm{H} \alpha$ are available for two other SNe IIn: SN 1997eg on day 198 (Salamanca et al. 2002) and SN 2002ic on day 256 (Kotak et al. 2004). For SN 1997eg the undisturbed CS velocity $u=20 \mathrm{~km} \mathrm{~s}^{-1}$ and preshock maximal velocity $u_{\text {max }}=160 \mathrm{~km} \mathrm{~s}^{-1}$ are infered from the $[\mathrm{Fe} \mathrm{X}] 6374 \AA$ and $\mathrm{H} \alpha$ profile modelling, whereas the forward shock velocity $v_{s}=5000 \mathrm{~km} \mathrm{~s}^{-1}$ is obtained from broad H $\alpha$ and He I $5876 \AA$ (Chugai 2019). Noteworthy, the CR acceleration efficiency reported in the latter paper is overestimated by a factor of two. In the case of SN 2002ic CS velocities $u=80 \mathrm{~km} \mathrm{~s}^{-1}$ and $u_{\max }=250 \mathrm{~km} \mathrm{~s}^{-1}$ are reported by Kotak et al. (2004), while the forward shock velocity $v_{s}=6000 \mathrm{~km} \mathrm{~s}^{-1}$ is obtained from the spectrum modelling (Chugai et al. 2004).

The efficiency of CR acceleration for three SNe IIn inferred using Equation (7) is given in Table 2. Surprisingly, all the values are close to each other within a factor of 1.5. This can be considered as a validation of the proposed diagnostics of the CR precursor of $\mathrm{SNe}$ IIn. On the other hand, the universality of the CR acceleration efficiency for three SNe IIn picked at random is not a trivial fact given a possible variation of environmental conditions.

\subsubsection{SN 2008iy radio emission}

The found CR acceleration efficiency can be applied to the interpretation of the SN 2008iy. The VLA observations at $8.46 \mathrm{GHz}$ (3.5 $\mathrm{cm}$ ) on 2009 April 24 (639 d after explosion) did not detect radio flux, whereas on 2009 December 26 (885 d) the radio was detected with the flux $f_{v} \approx 192 \pm 36 \mu \mathrm{Jy}$ (Chandra \& Soderberg 2009). This means that on day 639 the CSM was opaque to $f f$-absorption, while on day 885 CSM optical depth to $f f$-absorption was less then unity.

This conjecture can be verified using our model of the CSM and assuming that the $f f$-absorption is related to the intercloud CSM with the temperature of $T_{e}=10^{6} \mathrm{~K}$. The latter assumption is based on the presence of narrow coronal $[\mathrm{Fe} \mathrm{X}] 6374 \AA$ line in the latest spectrum on day 711 after discovery (Miller et al. 2010). The situation in this regard is similar to SN 1997eg (Chugai 2019). For SN 2008iy the calculated optical depth to $f f$-absorption at $\lambda=3.5 \mathrm{~cm}$ is $\tau_{f f}=4.05$ on day 639 and $\tau_{f f}=0.57$ on day 885 , which explains the evolution of radio flux between these two epochs. At the distance $D_{L}=179 \mathrm{Mpc}$ (Miller et al. 2010) the detected flux corresponds to the unabsorbed monochromatic luminosity $L_{v}=1.3 \times 10^{28} \mathrm{erg} \mathrm{s}^{-1} \mathrm{~Hz}^{-1}$.

The interaction model at this age suggests the CDS radius $r_{c d s}=$ $3.5 \times 10^{16} \mathrm{~cm}$, preshock density $\rho_{0}=5 \times 10^{-17} \mathrm{~g} \mathrm{~cm}^{-3}$, and the CDS velocity $v_{c d s}=2500 \mathrm{~km} \mathrm{~s}^{-1}$. The energy density of cosmic rays is $U_{c}=3 \epsilon \rho_{0} v_{s}^{2} \approx 0.36 \mathrm{erg} \mathrm{cm}^{-3}$ with $\epsilon \approx 0.038$. The energy density of relativistic electrons is $U_{e}=3.6 \times 10^{-3}\left(R_{e, p} / 0.01\right) \mathrm{erg} \mathrm{cm}^{-3}$, where $R_{e, p} \sim 10^{-2}$ is the typical fraction of the relativistic electron in the cosmic ray pressure produced largely by relativistic protons. For the power law distribution of relativistic electrons $d N / d E=K E^{-p}$ with $p=2$ the synchrotron emissivity (Getmantsev 1952) for the isotropic distribution of relativistic electrons is

$4 \pi j=4 \times 10^{-18} K \lambda^{1 / 2} B^{3 / 2} \mathrm{erg} \mathrm{s}^{-1} \mathrm{~cm}^{-3}$.

Adopting $E_{1}=1 \mathrm{MeV}$ and $E_{2}=10^{4} \mathrm{MeV}$ one gets $K=$ $U_{e} / \ln \left(E_{2} / E_{1}\right)=3.9 \times 10^{-4}\left(R_{e, p} / 0.01\right) \mathrm{erg} \mathrm{cm}^{-3}$. The volume of the radio-emittiing shell is $V=(4 \pi / 3) r^{3} \omega=9 \times 10^{49}(\omega / 0.5) \mathrm{cm}^{3}$ with $\omega \sim 0.5$ (e.g. Chevalier 1998) that corresponds to the shell thickness of $\Delta r=0.14 r_{c d s}$. The monochromatic radio luminosity at $\lambda=3.5 \mathrm{~cm}$ is thus $L_{v}=4 \pi j V=2.5 \times$ $10^{29} B^{3 / 2}(\omega / 0.5)\left(R_{e, p} / 0.01\right) \mathrm{erg} \mathrm{s}^{-1} \mathrm{~Hz}^{-1}$ which fits the observational luminosity for $B=0.14(\omega / 0.5)^{-2 / 3}\left(R_{e, p} / 0.01\right)^{-2 / 3} \mathrm{G}$. Interestingly, this value is only a factor of two lower comared to estimates for SN 1986J and SN 1988Z inferred using effects of the synchrotron self-absorption at low frequences (Chevalier 1998).

Moreover, the value $B \sim 0.14 \mathrm{G}$ is close to the saturated turbulent magnetic field predicted by the DSA theory. Indeed, the predicted energy density of the saturated turbulent magnetic field (Bell 2004) is

$U_{m} \sim 0.5\left(\frac{v_{s}}{c}\right) U_{c}$,

where $v_{s}$ is the forward shock speed, $c$ is speed of light. For SN 2008iy on day 885 with $v_{s} \approx v_{c d s}=2500 \mathrm{~km} \mathrm{~s}^{-1}$ and $U_{c}=0.36 \mathrm{erg} \mathrm{cm}^{-3}$ Equation (9) implies $B_{\text {sat }} \sim 0.19 \mathrm{G}$. The magnetic field in the radio-emitting shell of SN 2008iy thus coincides within a factor of unity with the saturated field suggested by the DSA mechanism. Note, for the estimated magnetic field of SN 2008iy the optical depth of the radio-emitting shell to the synchrotron selfabsorption on day 885 at $8.46 \mathrm{GHz}$ is small, $\tau_{s s a} \sim 0.14$, and therfore does nor affect our interpretation of radio emission.

To summarize, the recovered efficiency of cosmic ray acceleration in SN 2008iy combined with the CS interaction model is consistent with the radio emission of SN 2008iy for reasonable value of the magnetic field.

\section{DISCUSSION AND CONCLUSIONS}

The paper has been aimed at the interpretation of the optical phenomena related to the CS interaction of SN 2008iy. The bolometric light curve is found to be powered by the collision of the high energy $\left(3 \times 10^{51} \mathrm{erg}\right) \mathrm{SN}$ ejecta with the $\sim 10 M_{\odot} \mathrm{CS}$ envelope residing at the radius of $\sim 3 \times 10^{16} \mathrm{~cm}$. The maximal mass loss rate of presupernova is $\sim 0.09 \mathrm{M}_{\odot} \mathrm{yr}^{-1}$ that took place $\sim 200 \mathrm{yr}$ before the explosion. These estimates are based on the wind velocity $45 \mathrm{~km} \mathrm{~s}^{-1}$ 
inferred from the modelling of the CS $\mathrm{H} \alpha$ in the Keck I spectrum on day 711 (Miller et al. 2010).

The origin of enormous mass loss rate that closely precedes the explosion is puzzling. It looks like that the preSN envelope "knows" what is going on in the core either via pulsations caused by the hydrodynamic perturbations generated in the pre-collapse core (Shiode \& Quataert 2014) or, possibly, via a merger process that favours the vigorous mass loss and should end up with the core collapse.

While the progenitor nature is enigmatic, the large mass of CSM and enormous mass loss rate favour the massive progenitor with the main sequence mass probably exceeding $30 M_{\odot}$. In this context the former conjecture on the origin of SN $1988 \mathrm{Z}$ progenitor from stars in the range $8-10 M_{\odot}$ (Chugai \& Danziger 1994) requires revision. This reassessment is also supported by $8.5 \mathrm{yr}$-long observations of SN $1988 Z$ in the optical and X-ray band, which suggest enormous the radiation output $\sim 10^{51} \mathrm{erg}$ (for $H_{0}=70 \mathrm{~km} \mathrm{~s}^{-1} \mathrm{Mpc}^{-1}$ ) and the CS mass $\gg 1 M_{\odot}$ (Aretxaga et al. 1999). Noteworthy that the high explosion energy of SN 2008iy is on the verge of possibiities of the neutrino-driven mechanism that can provide $\lesssim 2 \times 10^{51} \mathrm{erg}$ (Janka 2017); the rotation energy of a collapsing core mediated by the magnetic field therefore is probably involved in the energetic explosion.

Based on the spectral similarity between SN 2008iy and SN $1988 Z$ emphasised by Miller et al. (2010) the luminous $\mathrm{H} \alpha$ line is attributed to the emission from CS clouds shocked and fragmented in the forward shock. The ionization of shocked clouds can be related to three sources: (i) X-rays from the forward shock,(ii) X-rays from cloud shocks, and (iii) extreme UV radiation from the boundary layer of cold dense fragments heated by the electron conductivity flux from the hot gas of the forwad shock. Without detailed modelling of the complex physics we are unable so far to rank these mechanisms according to their significance - all of them can contribute to $\mathrm{H} \alpha$ luminosity.

The $\mathrm{H} \alpha$ smootheness with the fluctuation amplitude $\lesssim 1 \%$ suggests that the number of line-emitting fragments of CS clouds in the forward shock should be $\gtrsim 10^{6}$. Although presumed, the fragmentation process of shocked clouds in SNe IIn has never been probed observationally. Here the fluorescent O I $8446 \AA$ emission line is used for the first time to demonstarate along with other observational constrants that the minimal length scale of line-emitting cloud fragments is 2.3 dex smaller compared to the minimal size of the undisturbed CS clouds. This should be considered as a convincing confirmation of the fragmentation cascade accompaniing the CS clouds disruption in the forward shock.

It should be admitted that the ejecta interaction with cloudy CSM is more complicated phenomenon than the presented scenario. The issue that requires elaborate study is the structure of the postshock layer between the forward shock and the CDS. The CDS Rayleigh-Taylor (RT) instability (Chevalier \& Blondin 1995; Blondin \& Ellison 2001) creates an extended boundary layer at the CDS interface with the forward shock filled in by the mixed RT spikes of a dense CDS material. In that case CS clouds or/and fragments may not be in time to completely mix in the postshock flow of the forward shock but instead interact with this dense boundary layer. At first glance this effect should not change significantly major results but for generality this process should be included in the detailed interaction scenario (e.g. Jun et al. 1996).

The analysis of the narrow $\mathrm{H} \alpha$ reveals the effect of preshock acceleration of CS gas identified with the outcome of CR precursor. The detection of this effect in CS H $\alpha$ of three random sample of SNe IIn (SN 2008iy, SN 1998eg, SN 2002ic) is of paramount significance by two reason. First, we acquire the simple efficient diagnostic tool to probe the CR acceleration efficiency in late time ( $2200 \mathrm{~d}$ ) SNe IIn based on high resolution $\left(\lesssim 50 \mathrm{~km} \mathrm{~s}^{-1}\right) \mathrm{H} \alpha$. Second, comparable values of the $\mathrm{CR}$ acceleration efficiency $(0.038,0.28,0.28)$ indicate weak sensitivity of $\epsilon$ value from environmental conditions in SNe IIn.

The synchrotron radio emission of SNe IIn, and young supernovae, in general, clearly demonstrates that the CS interaction is accompanied by the CR acceleration and magnetic field amplification (Chevalier 1982b, 1998). Note, however, that the radio data do not permit us to infer the CR ray acceleraton efficiency since the relativistic electrons is a minor constituent of the CR and their emissivity also depends on the unknown magnetic field. This emphasises the unique role of the diagnostics of the CR precursor based on the analysis of the resolved profile of CS $\mathrm{H} \alpha$ narrow line.

Noteworthy that the interaction model combined with the recovered efficiency of $\mathrm{CR}$ acceleration is consistent with the evolution of the radio emission of SN 2008iy, while the inferred magnetic field is compatible with the general prediction of the magnetic field amplification in the DSA theory.

\section{ACKNOWLEDGEMENTS}

I am grateful to Adam Miller for the Keck spectrum of SN 2008iy.

\section{DATA AVAILABILITY}

Details of the data analysis are available on request

\section{REFERENCES}

Aretxaga I., Benetti S., Terlevich R. J., Fabian A. C., Cappellaro E., Turatto M., della Valle M., 1999, MNRAS, 309, 343

Arnett W. D., 1980, ApJ, 237, 541

Axford W. I., Leer E., Skadron G., 1977, in International Cosmic Ray Conference. p. 132

Bell A. R., 2004, MNRAS, 353, 550

Blondin J. M., Ellison D. C., 2001, The Astrophysical Journal, 560, 244

Borkowski K. J., Shull J. M., 1990, ApJ, 348, 169

Bowen I. S., 1947, PASP, 59, 196

Capriotti E. R., 1965, ApJ, 142, 1101

Chandra P., Soderberg A., 2009, The Astronomer's Telegram, 2362, 1

Chandra P., Chevalier R. A., Chugai N., Milisavljevic D., Fransson C., 2020, ApJ, 902, 55

Chevalier R. A., 1982a, ApJ, 258, 790

Chevalier R. A., 1982b, ApJ, 259, 302

Chevalier R. A., 1998, ApJ, 499, 810

Chevalier R., Blondin J. M., 1995, The Astrophysical Journal, 444, 312

Chugai N. N., 1990, Soviet Astronomy Letters, 16, 457

Chugai N. N., 1993, ApJ, 414, L101

Chugai N. N., 2018, MNRAS, 481, 3643

Chugai N. N., 2019, Astronomy Letters, 45, 71

Chugai N. N., Danziger I. J., 1994, MNRAS, 268, 173

Chugai N. N., Chevalier R. A., Lundqvist P., 2004, MNRAS, 355, 627

Cowie L. L., McKee C. F., 1977, ApJ, 211, 135

Fassia A., et al., 2001, MNRAS, 325, 907

Filippenko A. V., 1991, in Woosley S. E., ed., Supernovae. p. 467

Getmantsev G. G., 1952, Akademiia Nauk SSSR Doklady, 83, 557

Hansen J. F., Robey H. F., Klein R. I., Miles A. R., 2007, ApJ, 662, 379

Janka H.-T., 2017, Neutrino-Driven Explosions. p. 1095, doi:10.1007/978-3-319-21846-5_109

Judge P. G., Neff D. H., 1990, in Wallerstein G., ed., Astronomical Society of the Pacific Conference Series Vol. 9, Cool Stars, Stellar Systems, and the Sun. p. 57 
Jun B.-I., Jones T. W., Norman M. L., 1996, ApJ, 468, L59

Khazov D., et al., 2016, ApJ, 818, 3

Klein R. I., McKee C. F., Colella P., 1994, ApJ, 420, 213

Klein R. I., Budil K. S., Perry T. S., Bach D. R., 2003, ApJ, 583, 245

Kotak R., Meikle W. P. S., Adamson A., Leggett S. K., 2004, MNRAS, 354, L13

Kozasa T., Hasegawa H., Nomoto K., 1989, ApJ, 344, 325

Krymskii G. F., 1977, Akademiia Nauk SSSR Doklady, 234, 1306

Leibundgut B., Kirshner R. P., Pinto P. A., Rupen M. P., Smith R. C., Gunn J. E., Schneider D. P., 1991, ApJ, 372, 531

Maeda K., et al., 2013, ApJ, 776, 5

Martin P. G., 1988, ApJS, 66, 125

Miller A. A., et al., 2010, MNRAS, 404, 305

Netzer H., Penston M. V., 1976, MNRAS, 174, 319

Pozzo M., Meikle W. P. S., Fassia A., Geballe T., Lundqvist P., Chugai N. N., Sollerman J., 2004, MNRAS, 352, 457

Rupen M. P., van Gorkom J. H., Knapp G. R., Gunn J. E., Schneider D. P., 1987, AJ, 94, 61

Salamanca I., Terlevich R. J., Tenorio-Tagle G., 2002, MNRAS, 330, 844

Schlegel E. M., 1990, MNRAS, 244, 269

Shiode J. H., Quataert E., 2014, ApJ, 780, 96

Smith N., McCray R., 2007, ApJ, 671, L17

Stathakis R. A., Sadler E. M., 1991, MNRAS, 250, 786

Sutherland R. S., Dopita M. A., 1993, ApJS, 88, 253

Weiler K. W., Panagia N., Sramek R. A., 1990, ApJ, 364, 611

Woosley S. E., Blinnikov S., Heger A., 2007, Nature, 450, 390

Xu Y., McCray R., Oliva E., Rand ich S., 1992, ApJ, 386, 181

Zhang T., et al., 2012, AJ, 144, 131

This paper has been typeset from a $\mathrm{T}_{\mathrm{E}} \mathrm{X} / \mathrm{L} \mathrm{AT} \mathrm{E}$ file prepared by the author. 